\title{
The Effects of Strabismus Surgery on Anterior Segment Circulation
}

\author{
J. M. OLVER and J. P. LEE
}

London

\begin{abstract}
Summary
Anterior segment circulation was assessed in 35 adults one day after squint surgery by clinical observation and low-dose fluorescein iris angiography. Seventeen patients had primary vertical rectus muscle surgery and all showed angiographic evidence of ischaemia. No ischaemia was found in the $\mathbf{1 5}$ patients who had secondary vertical rectus muscle surgery, or any horizontal rectus muscle surgery. The staged group had intermediate findings between the above two. Age, dysthyroid eye disease and type of conjunctival incision did not correlate with fluorescein iris angiographic sector-filling delay on the first post-operative day. The time taken for the sector with delay to fill becomes less during the first two post-operative weeks. Redistribution of iris filling persists, however. This data suggest that the safe interval before further muscle surgery can be done is shorter than has previously been assumed. Since the anterior ciliary arteries do not reform into canals the probable mechanism of redistribution of blood flow is from the long posterior ciliary arteries and increased capacity of the collateral circulation.
\end{abstract}

The number of muscles which may be operated upon in adults with strabismus is limited by the risk of ischaemia. This may result from simultaneous surgery on three rectus muscles in healthy adult patients ${ }^{1,2,3,4}$ or even two rectus muscles in unhealthy patients such as those with chronic lymphocytic leukemia $^{5}$ and dysthyroid eye disease ${ }^{6}$ and may lead to anterior segment ischaemia. It has been documented occasionally in children ${ }^{3,7}$ in whom there were significant associated ocular abnormalities (e.g. congenital absence of three muscles, severe retinopathy of prematurity.) The incidence of ischaemia following strabismus operations for all age groups is estimated to be one in $13,333 .{ }^{3}$ Anterior segment ischaemia rarely leads to blindness but may result in reduced vision and symptoms of glare due to iris atrophy and corectopia. The risk however is sufficiently significant to restrict surgery to moving only two rectus muscles at any one operation. ${ }^{1,2,3,8}$ Any further rectus muscle surgery is staged two to three months later ${ }^{2,3,8}$ to allow for restoration of blood flow in the anterior segment. Botulinum toxin may be used as an alternative to surgery to produce chemical paralysis of the medial rectus, allowing safe two muscle surgery. ${ }^{2,9}$

In this study we investigated the early sequelae of strabismus surgery on the iris circulation. The mechanism of restoration of blood flow and the clinical implications are discussed. 


\section{Methods}

Thirty five adult patients undergoing strabismus surgery at Moorfields Eye Hospital under the care of Mr John Lee and $\mathrm{Mr}$ Peter Fells were studied. Since stromal pigment in brown irides obscures the view of fluorescein in the iris vessels, only patients with blue eyes were included. Patients with a history of drug allergy or atopy were excluded.

Pre-operative assessment included clinical and slit-lamp examination, orthoptic assessment, anterior segment colour photography and low-dose fluorescein iris angiography. We used low dose fluorescein which has the benefit of also visualising the episcleral vessels as described by Meyer. ${ }^{10}$ Thyroid function tests were done if indicated and a record made of systemic disease. Surgical details including type of conjunctival incision were noted.

On the first post-operative day (12-18 hours post-operatively) the degree of any acute anterior segment ischaemia was assessed by clinical and angiographic examination. The anterior segment was examined for functional signs of ischaemia; corectopia, irregular pupil reaction in the segment corresponding to angiographic delay, presence of uveitis and striate keratitis. Low-dose fluorescein iris angiography was repeated.

The degree of ischaemia was graded according to severity and recorded. This ranged from Grade 1, simple angiographic sector-filling delay alone, Grade 4 , overt sector ischaemia. (Table I).

Normal iris filling in man (the time taken for complete filling to the pupillary border from the first appearance of fluorescein in iris arterioles) is under 10 seconds. ${ }^{11}$ Sector filling delay exceeding this time is regarded as abnormal. (Table II).

The patients were grouped according to the type of surgery for statistical analysis, ie whether the surgery was primary, secondary, subsequent (staged) and whether it involved vertical or horizontal rectus muscles. Primary rectus muscle surgery was distinguished from secondary rectus muscle surgery. Secondary surgery denotes surgery to a previously operated muscle. Staged rectus muscle surgery denotes surgery to previously unoperated muscles where other rectus muscles in the same eye have been operated on. If sector filling delay was present on Day 1 , the patient was entered into a longitudinal study and followed up at regular intervals for clinical examination and repeat iris angiography.

\section{Photographic methods}

Low dose iris angiography using $0.6 \mathrm{ml}$ of sodium fluorescein 20 per cent was carried out using standard anterior segment photographic equipment. This comprised a Zeiss (Oberkocken) photoslit lamp (flash power output of $720 \mathrm{w} / \mathrm{s}$ and recycling time $1-1.5 \mathrm{~s}$ ) with an attached Contax RTS-II camera with a Dataphot back, using a Spectrotech exciter filter and Wratten 12 barrier filter. A $\times 2$ converter and slit-lamp magnification of $\times 10$ gave a magnification at the camera back of $\times 2$. Kodak T-Max 400 film was used and was developed in T-Max 400 developer (1:4) for 9 minutes at $20^{\circ} \mathrm{C}$.

Table I Clinical grading of acute anterior segment ischaemia

\begin{tabular}{lll}
\hline Grade & Signs & Symptoms \\
\hline 1. Sector delay. & Angiographic sector-filling delay. See Table II. & Asymptomatic \\
2. Pupil dysfunction & $\begin{array}{l}\text { Angiographic sector-filling delay associated with: } \\
\text { Corectopia, irregular pupil reaction and flattening of the } \\
\text { pupil margin. Transient slit lamp examination finding. }\end{array}$ & Asymptomatic \\
& $\begin{array}{l}\text { Uveitis in the early post-operative phase. Pupil signs are } \\
\text { more marked and may be visible macroscopically. Iris } \\
\text { 3. Vascular leakage }\end{array}$ & $\begin{array}{l}\text { Symptomatic } \\
\text { (Photophobic) }\end{array}$ \\
& $\begin{array}{l}\text { Striate keratitis, uveitis, decreased intra-ocular pressure } \\
\text { and deepened anterior chamber. 21 Glaukomflecken. } \\
\text { 4. Infarction }\end{array}$ & $\begin{array}{l}\text { Symptomatic } \\
\text { (Reduced vision, }\end{array}$ \\
& Sector infarction approx.=>270 o. Marked iris atrophy. & pain) \\
\hline
\end{tabular}


Table II Definition of sector filling delay

1. A sector delay with an angle exceeding $35^{\circ}$ or over one clock hour.

2. The duration of the delay, the time taken for the sector to completely fill from the first appearance of fluorescein in the iris vessels, exceeds 10 seconds. ${ }^{11,18,20}$

3. The delay in that sector was not previously present.

4. The delay is a repeatable finding which persists

Table III Indications for surgery $n=35$

\begin{tabular}{lcc}
\hline Strabismus type & no. & $\%$ \\
\hline $\begin{array}{l}\text { Concomitant } \\
\text { Paralytic } \\
\quad \text { (Third nerve palsy 1, } \\
\quad \text { fourth nerve palsy 4, } \\
\quad \text { sixth nerve palsy 5) }\end{array}$ & 15 & 45 \\
$\begin{array}{l}\text { Restrictive } \\
\quad \text { Dysthyroid eye disease 6, }\end{array}$ & 10 & 28 \\
$\quad$ Orbital trauma 2) & 8 & \\
Other & 22 \\
\hline
\end{tabular}

\section{Results}

The indications for surgery are summarised in Table III. The operation details are summarised in Table IV, the fluorescein iris angiography findings according to the type of surgery in Table $\mathrm{V}$ and clinical grading of anterior segment ischaemia in Table VI.

Surgery was on one or two rectus muscles in all patients except those who had subsequent (staged) surgery, in whom all four rectus muscles were operated upon.

There were 17 female and 18 male patients with an age range of 17-80 years (mean age 40 years, standard deviation 16.2). Patients were grouped by age for analysis; those 45 years and less and those 46 years and over. Follow up ranged from two to nine months. Twenty four patients had primary rectus muscle surgery and eight patients had secondary rectus muscle surgery. Three patients had subsequently (staged) rectus muscle surgery.

All patients who underwent primary surgery to vertical rectus muscles (17 patients) had angiographic sector-filling delay on Day 1 (Figs 1-4). In these patients with sector-filling delay, iris filling ranged from 25 to 90 seconds (mean 38 seconds, median 36 seconds), which represents a true delay exceeding $25-80$ seconds. No sector-filling delay was evident in the 15 patients who had either secondary vertical rectus muscle surgery or primary or secondary horizontal rectus muscle surgery. Of three patients who had subsequent (staged) surgery, one showed no evident sector filling delay, whilst two did (Table V).

Six of the primary vertical rectus muscle surgery patients with angiographic evidence of ischaemia also showed clinical signs of anterior segment ischaemia, Grades 2 and 3. (Table VI). These patients included both dysthyroid and non-dysthyroid patients. None had Grade 4 acute anterior segment ischaemia (sector infarction).

The type of conjunctival incision (Fig. 5) was not associated with angiographic sector filling delay.

Increased age was associated with angiographic sector-filling delay on the first post-operative day in the whole group (Table VIIa) but not in those patients undergoing

Table IV Operations $n=35$

\begin{tabular}{llrr}
\hline & $\begin{array}{l}\text { Rectus } \\
\text { muscles }\end{array}$ & no. & $\%$ \\
\hline Single muscle & SR & 2 & 6 \\
& IR & 11 & 31 \\
Non-adjacent muscles & SR IR & 7 & 20 \\
& MR LR & 13 & 37 \\
Adjacent muscles & MR IR & 2 & 6 \\
\hline
\end{tabular}

Table $\mathbf{V}$ Type of Surgery and presence of sector delay on day one $n=35$

\begin{tabular}{llrrc}
\hline & & no. & $\%$ & Delay \\
\hline Primary & vertical & 15 & 43 & All \\
& vert+adj & 2 & 6 & All \\
& horizontal & 7 & 20 & None \\
Secondary & vertical & 3 & 9 & None \\
& horizontal & 5 & 14 & None \\
Subsequent & vert to horiz & 2 & 6 & One \\
& horiz to vert & 1 & 2 & One \\
\hline
\end{tabular}

Table VI Clinical grading acute ASI $\quad n=19$

\begin{tabular}{lcc} 
Table VI Clinical grading acute & ASI & $n=19$ \\
\hline & $n o$. & $\%$ \\
\hline 1. Sector delay & 13 & 67 \\
2. Pupil dysfunction & 4 & 21 \\
3. Vascular leakage & 2 & 11 \\
4. Infarction & 0 & 0 \\
\hline
\end{tabular}




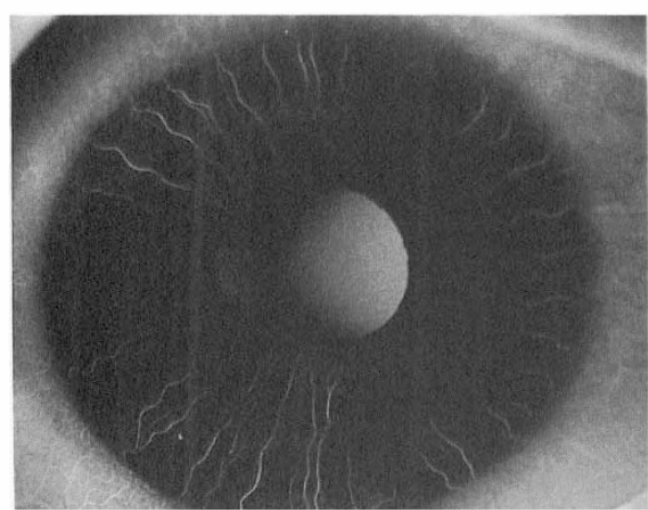

Fig. 1a

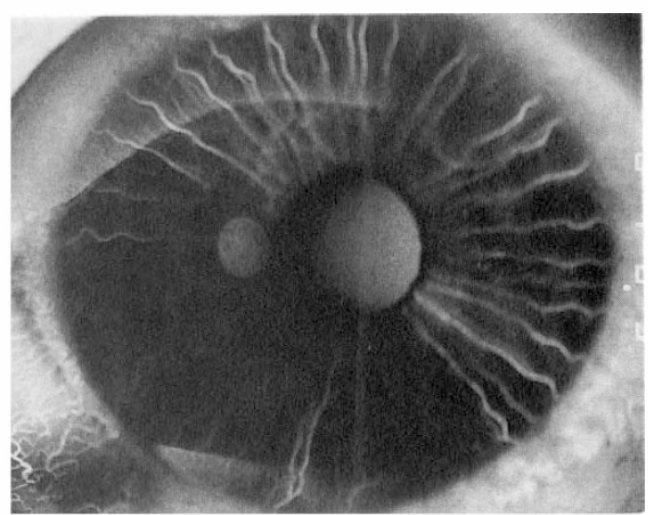

Fig. $1 b$

Fig. 1 Right primary inferior rectus recession. 19 year old male. (a) Pre-operative. Normal iris angiogram, midarterial phase. (b) First day post-operative. Infero-temporal sector-filling delay, corresponding mid-arterial phase.

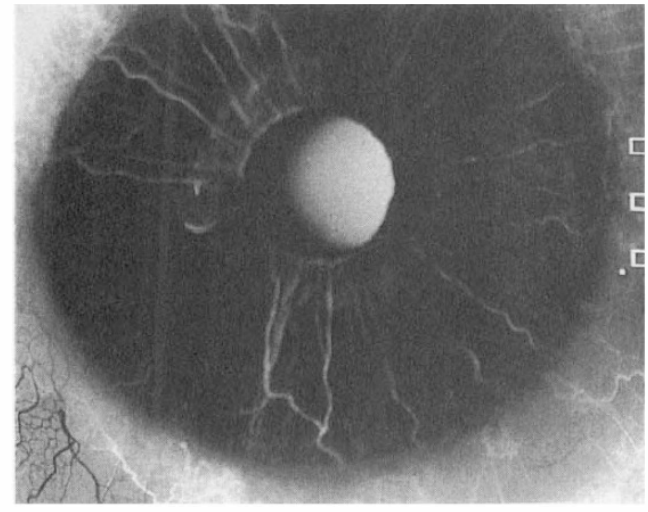

Fig. 2a

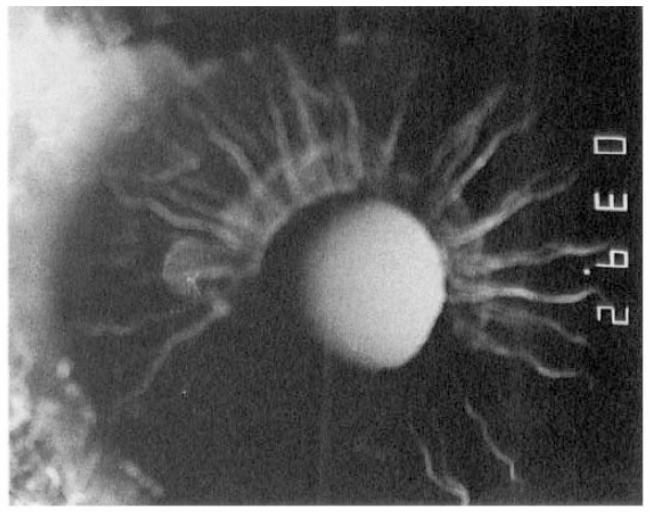

Fig. 2b

Fig. 2 Right primary inferior and medial rectus recession (dysthyroid patient). 49 year old male. (a) Preoperative. Normal iris angiogram, mid-arterial phase. (b) First day post-operative. Infero-temporal sector-filling delay, late arterial phase.

primary vertical rectus muscle surgery (Table VIIb). These tables include two patients with subsequent (staged) primary vertical rectus muscle surgery.

The effect of dysthyroid eye disease was significantly associated with the presence of angiographic sector-filling delay on the first post-operative day when analysed for all types of surgery (see Table VIIIa). This effect was lost when analysed within those patients who had primary vertical rectus muscle surgery (see Table VIIIb). The six patients with dysthyroid eye disease all had primary vertical rectus muscle surgery. The type of surgery was the determining factor (see Table IX).

\section{Discussion}

Hayreh and Scott reported angiographic iris sector-filling defects following vertical rectus muscle surgery in seven eyes. ${ }^{12}$ This prospective study demonstrates that the type of surgery is the most important factor determining anterior segment ischaemia. Primary vertical rectus muscle surgery caused sector filling delay on the first post-operative day in all patients except one who had subsequent (staged) surgery. This finding is not present after secondary vertical rectus muscle surgery or any type of horizontal rectus muscle surgery, either primary or secondary.

Our study demonstrates that primary 


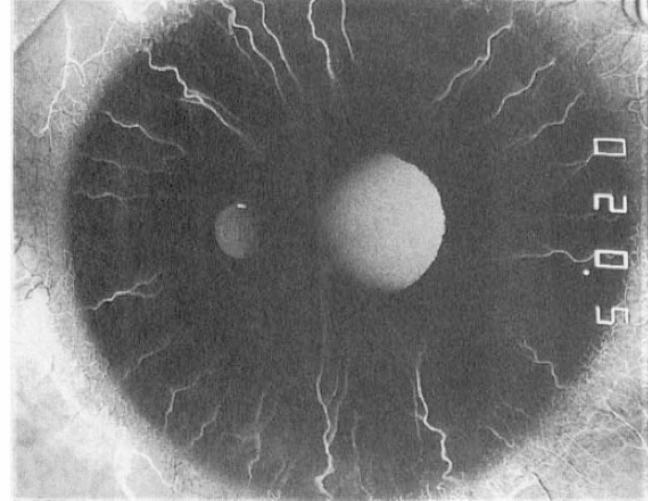

Fig. 3a

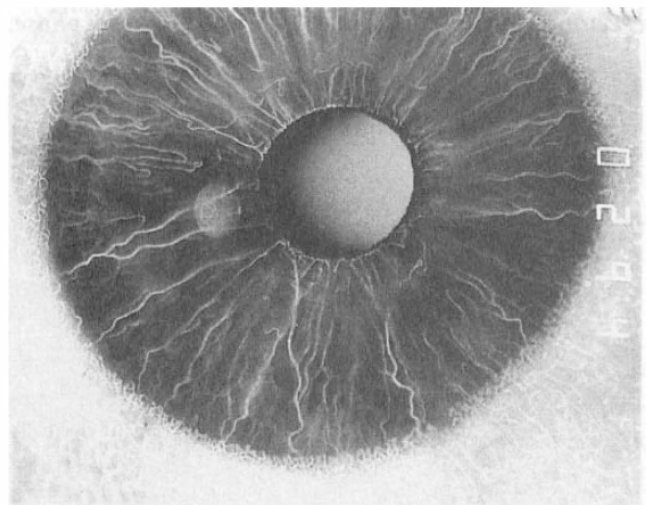

Fig. 3b

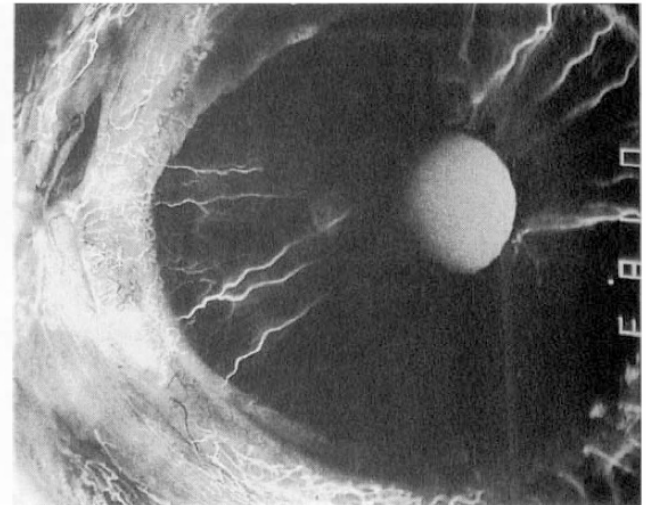

Fig. 3c

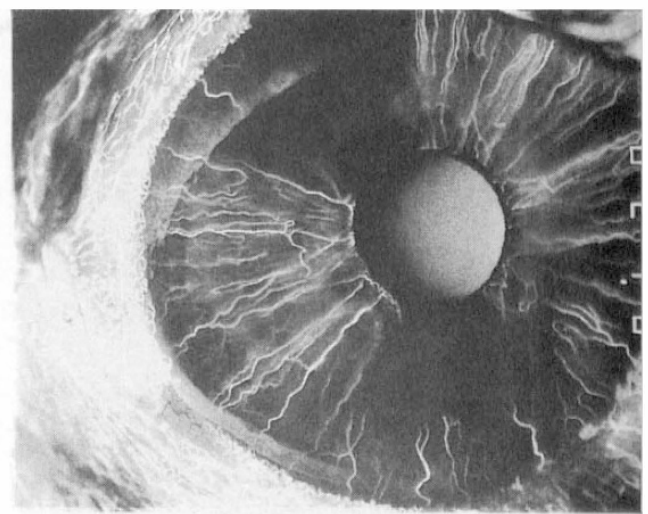

Fig. 3d

Fig. 3 Right primary superior and inferior rectus transposition. 30 year old male. $(a, b)$ Pre-operative. Normal iris angiograms. Mid and late arterial phases. (c,d) First day post-operative. Superior and inferior sector-filling delay. Corresponding mid and late arterial phases.

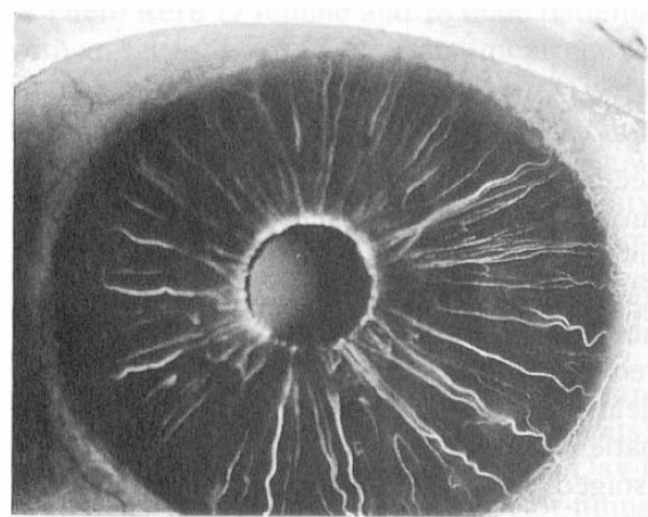

Fig. 4a

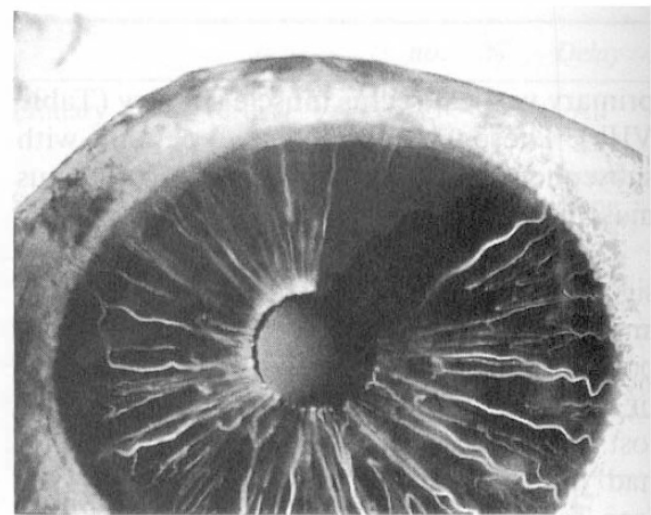

Fig. $4 b$

Fig. 4 Left primary superior rectus recession. 55 year old female. (a) Pre-operative. Normal iris angiogram. Late arterial phase. (b) First day post-operative. Superior sector-filling delay. Corresponding late arterial phase. 
Table VIIa Sector filling delay Day 1: Association of age following all types of surgery

$n=35$

\begin{tabular}{ccc}
\hline & Delay present & Delay absent \\
\hline Age in years & & \\
$<45$ & 8 & 14 \\
$>46$ & 11 & 2 \\
& & 2 \\
& $\mathrm{x}=46.24$ & $\mathrm{p}<0.001$ \\
\hline
\end{tabular}

Table VIIb Sector filling delay Day 1: Association of age following primary vertical rectus muscle surgery $n=19$

\begin{tabular}{ccc}
\hline & Delay present & Delay absent \\
\hline Age in years & & \\
$<45$ & 8 & 1 \\
$>46$ & 10 & 0 \\
& Fisher exact test NS \\
\hline
\end{tabular}

inferior rectus muscle surgery resulted in a larger area with delay (Fig. 1) than following primary superior rectus tenotomy (Fig. 4). There is no apparent difference in the size of the sector with delay between a single inferior rectus tenotomy (Fig. 1) and combined inferior and medial rectus tenotomy, (Fig. 2). Hayreh and Scott ${ }^{11}$ suggested that combined lateral and inferior rectus tenotomies (based on data from one patient, two eyes) caused a larger effect on the iris circulation than inferior and medial rectus tenotomy. They concluded that the anterior ciliary artery from the lateral rectus contributed more than those from the medial rectus. The evidence against this is anatomical. Anterior segment vascular casting $^{13,14}$ in non-human primates and in humans show that the single lateral anterior ciliary artery does not supply the intra-muscular arterial circle and hence iris, but anterior episleral arterial circle and the ciliary body.

Angiographically a lateral rectus tenotomy does not cause sector filling delay and since the sector-delay following vertical tenotomy is always temporally placed, it can be assumed that the small strip of iris temporally is supplied by the lateral long posterior ciliary artery.

Medially the iris blood supply is derived partly from the long posterior ciliary artery, which masks flow derived from the anterior ciliary arteries. Hence any evident sector delay following medial rectus tenotomy is obscured. In addition, experimental evidence from radio-nucleotide labelled microsphere studies $^{15,16}$ show that immediately following rectus muscle tenotomy there is a compensatory increase in blood flow attributed to the long posterior ciliary arteries. Therefore following primary horizontal rectus muscle surgery blood flow from the medial long posterior ciliary artery prevents any delay in filling being evident horizontally. The microsphere experiments also demonstrated a greater increase in blood flow from the medial than from the lateral long posterior ciliary artery, which may also account for the temporally placed sector-filling delay following vertical rectus muscle surgery.

In contrast to primary vertical rectus muscle surgery, secondary vertical rectus muscle surgery did not cause sector filling delay. France ${ }^{17}$ suggested that re-establishment of blood flow through the tenotomised rectus muscle occurs and quoted anecdotal evidence that the

Table VIIIa Sector filling delay Day 1: Association of dysthyroid eye disease following all types of surgery $n=35$

\begin{tabular}{lcc}
\hline & Delay present & Delay absent \\
\hline Dysthyroid & 6 & 0 \\
Non-dysthyroid & 13 & 16 \\
& & 2 \\
& $\mathrm{x}=12.95$ & $\mathrm{p}<0.01$ \\
\hline
\end{tabular}

Table VIIIb Sector filling delay Day 1: Association of dysthyroid eye disease following primary vertical rectus muscle surgery $n=19$

\begin{tabular}{lcc}
\hline & Delay present & Delay \\
\hline Dysthyroid & 6 & 0 \\
Non-dysthyroid & 12 & 1 \\
& Fisher exact test NS \\
\hline
\end{tabular}

Table IX Sector filling delay Day 1: Association of type of surgery in non-dysthyroid patients $\quad n=29$

\begin{tabular}{lcc}
\hline & Delay present & Delay absent \\
\hline Primary vertical & 13 & 0 \\
All other & 1 & 15
\end{tabular}

Fisher exact probability test $\mathrm{p}<0.0001$ 
a

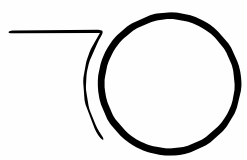

C
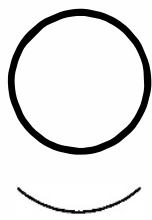

d

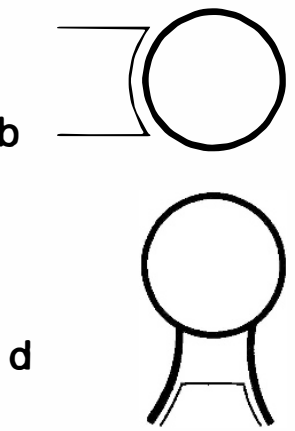

Fig. 5 Types of conjunctival incisions Horizontal rectus muscle surgery, (a) triangular fornix based, (b) quadrilateral fornix based. Vertical rectus muscle surgery, (c) over muscle insertion, (d) quadrilateral fornix based with conjunctival recession.

anterior ciliary arteries are recanalised. Three observations make this seem unlikely.

First, secondary vertical rectus muscle surgery does not cause sector filling delay. Secondly, episceral angiography shows no evidence of blood flow across the strabismus scar (unpublished data). Thirdly, the redistribution of blood flow following vertical rectus muscle surgery is permanent; the sector with delay remaining the last to fill. These permanent alterations in filling patterns can be seen clearly using video-angiography.

The type of conjunctival incision ${ }^{2.12,18}$ (Fig. 5) has also been suggested as being important in predisposing to anterior segment ischaemia, by damaging the anterior episcleral arterial circle (A E A C). The A E A C is an important collateral arterial circle which links adjacent anterior ciliary arteries and has numerous perforating branches to the intramuscular arterial circle, (Fig. 6), but is unlikely to be damaged during strabismus surgery since it lies in the deepest episcleral layer and is therefore not damaged when the conjunctiva is reflected. Extensive damage to the A E A C may precipitate anterior segment ischaemia if the circulation is compromised.

Sector filling delay is defined as in Table II. More than $270^{\circ}$ of sector delay is required for sector infarction. It is important not to confuse an anomalous iris circulation with sectorfilling delay. If the major arterial circle of the iris loops towards the pupil, an apparent area of delayed fill is seen within its concavity. However, the sigmoid curve at or close to the pupil margin differentiates these two conditions (Fig. 7).

Adjacent episcleral vascular delay is also evident following primary vertical rectus muscle surgery. This initially has a similar delay to that of the iris but on subsequent angiograms the delay is reduced. This represents the immediate contribution by the anterior ciliary arteries to the episclera and conjunctiva.

The term anterior segment ischaemia has been used in the literature to describe a range of ischaemia from simple sector filling delay seen on fluorescein angiography to overt sector infarction. We therefore used a grading system based on other authors' and our own observations to help illustrate the range of ischaemia and to standardise interpretation of results.

The findings in the small number of patients with subsequent (staged) surgery support the observation of Hayreh from primate studies that staged surgery reduces the effect on the iris circulation..$^{12}$ These patients all had staged four rectus muscle surgery. Of the two patients with previous horizontal rectus tenotomies, only one aged 17 years developed evident sector-filling delay following primary vertical rectus tenotomies, even though there were 10 years between operations. The other (aged 20 years), with a surgical interval of two years did not. One patient aged 50 years had horizontal tenotomies and developed exten-

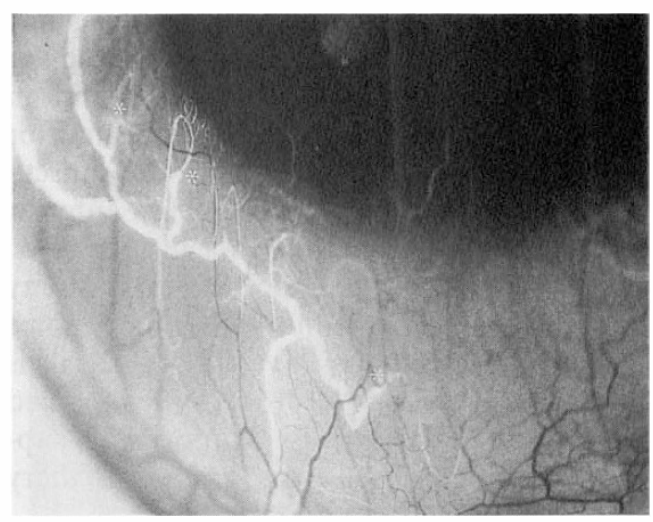

Fig. 6 Low dose fluorescein angiogram right inferotemporal episclera two months following inferior rectus recession showing part of the anterior episcleral arterial circle linking inferior and lateral anterior ciliary artery territory; points of perforating arteries denoted by * 


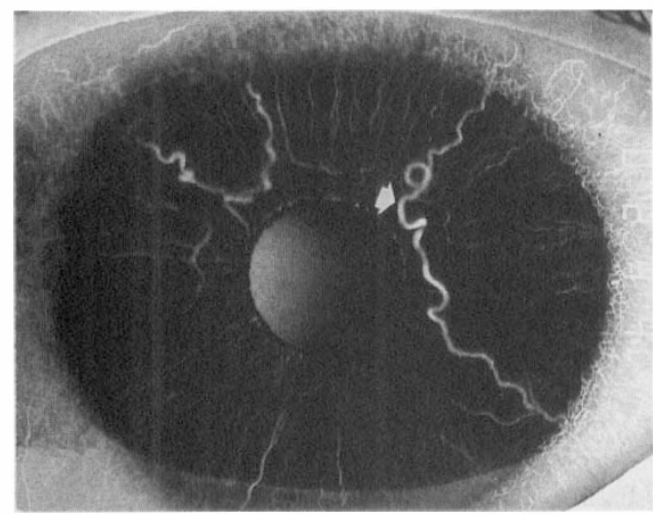

Fig. 7 Right iris angiogram demonstrating obvious anomalous arterial circulation from iridial placed major arterial circle. Characteristic sigmoid shape vessel at pupillary margin, see arrow.

ANTERIOR SEGMENT CIRCULATION BEFORE \& AFTER VERTICAL SQUINT SURGERY

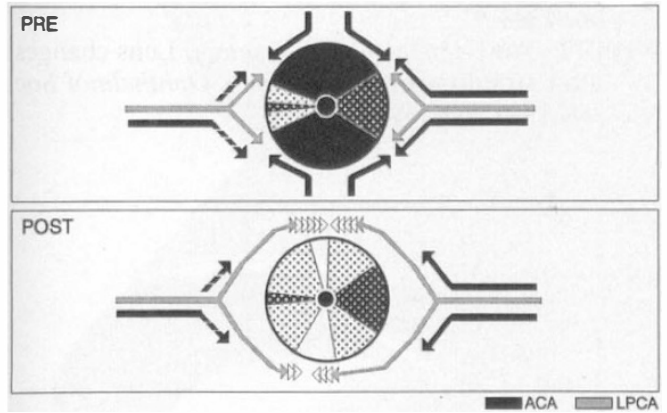

Fig. 8 Diagram of right iris blood supply pre-and post- primary vertical rectus muscle surgery, illustrating relative changes in distribution of anterior and posterior ciliary artery supply. In adults there are inadequate compensatory mechanisms, resulting in evident sector filling delay.

sive evident delay superiorly and inferiorly. This was either due to inadequate compensation from the long posterior ciliary arteries or capacity of the collateral arterial circles, resulting in evident sector delay in the most vulnerable zones.

The preliminary results of the outcome of sector-filling delay show that the time taken for the sector with delay to fill rapidly reduces over the first two weeks, from a range of 25 to 90 seconds on the first day, to a plateau which is often slightly longer than the accepted normal iris filling time of 6-10 seconds, at for instance 12-15 seconds; whilst the size of the sector remains the same. The change in filling pattern after surgery persists; the sector with delay remaining the last to fill. This rapid reduction in delay over the first two weeks to near or within normal filling times, suggests that the safe interval may be shorter than the assumed two or three months before further staged surgery. The almost complete absence of sector infarction in children compared to adults suggests that the latter do not have a sufficiently adaptable vascular system to prevent a noticeable sector with delay, (see Fig. 8).

There was no association of increased age nor of dysthyroid eye disease with the development of sector filling delay on Day 1 . It is not possible to differentiate dysthyroid patients from the non-dysthyroid patients, since the dysthyroid patients all had primary vertical rectus muscle surgery which itself results in sector delay. A larger number of patients is required satisfactorily to analyse the severity of ischaemia on the first postoperative day and the outcome of ischaemia for the two groups.

In summary, it is recommended that when strabismus surgery is planned the type of surgery is taken into account, whether primary or secondary, vertical or horizontal. Routine pre-operative angiograms are not recommended. It is safer to stage surgery; the safe time interval before further surgery may be shorter than was previously thought. If in doubt, botulinum toxin should be considered, either as an adjunct or as primary therapy.

We would like to thank Mr Peter Fells for allowing us to study his patients, who formed a large proportion of this study, and the Medical Illustration Department at Moorfields Eye Hospital particularly Annie Bolton and Max Browne for technical assistance.

\section{References}

1 Von Noorden GK: Anterior segment ischaemia following the Jensen procedure. Arch Ophthalmol 1976, 94: 845-7.

2 Saunders RA and Sandell GS: Anterior segment ischaemia syndrome following rectus muscle transposition. Am J Ophthalmol 1982, 93: 34-8.

${ }^{3}$ France TD and Simon JW: Anterior segment ischaemia syndrome following muscle surgery: the AAPO\&S experience. $J$ Paed Ophthalmol Strabismus 1986, 23: 87-91.

${ }^{4}$ Frey T: Anterior segment ischaemia caused by Jensen's procedure. J Ocul Ther Surg 1985, 3: 242-5

${ }^{5}$ Jacobs DS, Vastine DW, Urust MJ: Anterior segment ischaemia and sector iris atrophy: after 
strabismus surgery in a patient with chronic lymphocytic leukaemia. Ophthal Surg 1976, 7: 42-8.

${ }^{6}$ Fells P and Marsh RJ: Anterior segment ischaemia following surgery on two rectus muscles. In: Strabismus. Ed. R D Reinecke. 375-80 1978.

${ }^{7}$ Elsas FJ and Witherspoon CD: Anterior segment ischaemia after strabismus surgery in a child. $\mathrm{Am}$ J Ophthalmol 1987, 103: 833-4.

${ }^{8}$ Simon JW, Price EC, Krohel GB, Poulin RW, Reinecke RD: Anterior segment ischaemia following strabismus surgery. J Paed Ophthalmol Strabis 1984, 21: 179-84.

${ }^{9}$ Fitzsimons R, Lee J, Elston J: Combined therapy of sixth nerve palsey with surgery and botulinum toxin chemodenervation. Ophthalmology 1988, 95: $1535-42$.

${ }^{10}$ Meyer PAR and Watson PG: Low dose fluorescein angiography of the conjunctiva and episclera. $\mathrm{Br} \mathrm{J}$ Ophthalmol 1987, 71: 2-10.

11 Van Nerom PR, Rosenthal AR, Jacobson DR, Pieper I, Schwartz H, Grieder BW: Iris angiography and aqueous photofluorometry in normal subjects. Arch Ophthalmol 1981, 99: 489-93.

${ }^{12}$ Hayreh SS and Scott WE: Fluorescein iris angiography. II. Disturbances in iris circulation following strabismus operation on the various recti. Arch Ophthalmol 1978, 96: 1390-400.
${ }^{13}$ Morrison JC and Van Buskirk EM: Anterior collateral circulation in the primate eye. Ophthalmol 1983, 90: 707-15.

14 Woodlief NF: Initial observations on the ocular microcirculation in man. I. The anterior segment and extraocular muscles. Arch Ophthalmol 1980, 98: $1268-72$.

15 Wilcox LM, Keough EM, Connolly RJ: Regional ischaemia and compensatory vascular dynamics following selective tenotomy in primates. Exp Eye Res 1981, 33: 353-60.

${ }^{16}$ Keough ' EM, Connolly R, Ramberg-Laskaris K, Wilcox LM: Primate ocular blood flow following contiguous recti tenotomy. Ophthalmic Res 1983, 15: 204-7.

${ }^{17}$ France TD: In Discussion (Ref 3) J Paed Ophthalmol Strabis. 1984, 21: 185.

18 Virdi PS and Hayreh SS: Anterior segment ischaemia after recession of various recti. An experimental study. Ophthalmol 1987, 94: 1258-70.

${ }^{19}$ Hayreh SS and Scott WE: Fluorescein iris angiography. I. Normal pattern. Arch Ophthalmol 1978, 96: 1383-9.

${ }^{20}$ Fells P: Anterior segment ischaemia. Lens changes after strabismus surgery. Trans Ophthalmol Soc UK 1980, 100: 398-9. 